\title{
Three TMS Members Selected for National Academy of Engineering
}

\section{member news}

Share the good news about your professional accomplishments! Contact Kaitlin Calva, JOM Magazine Managing Editor, at kcalva@tms.org. Please note that only news submitted by current TMS members will be considered.

\section{TMS Members Elected to NAE}

Congratulations are in order for three TMS members who were elected to the U.S. National Academy of Engineering (NAE) this year. Election to the NAE is among the highest professional distinctions for engineers and honors those who have made outstanding contributions to "engineering research, practice, or education, including, where appropriate, significant contributions to the engineering literature" and to "the pioneering of new and developing fields of technology, making major advancements in the traditional fields of engineering, or developing/implementing innovative approaches to engineering education."

The following TMS member will be inducted as one of 87 U.S. members in the NAE:

\section{Paul E. Krajewski}

Krajewski is the director, global research and development, at General Motors Co., in Warren, Michigan. He was elected "for development and implementation of lightweight automotive materials." A TMS member since 1993, Krajewski received the 2013 AIME Champion H. Matthewson Award and was in the first class of TMS Brimacombe Medalists in 2012.

The following TMS members will be inducted as two of 18 international members in the NAE:

\section{Eduard Arzt}

Arzt, the chief executive officer and scientific director at INM-Leibniz Institute for New Materials of Saarland University in Saarbrücken, Germany, was elected "for research on mechanical properties and development of bio-inspired functional surfaces for medical adhesives and novel gripping systems." A longtime member of TMS, Arzt is the 2020 recipient of the TMS Morris Cohen Award.

\section{Chennupati Jagadish}

A distinguished professor of electronic materials engineering at the Australian National University in Canberra, Jagadish was elected "for contributions to nanotechnology for optoelectronic devices." He has been a TMS member since 2013 and is also a 2015 Fellow of the U.S. National Academy of Inventors.

The new class will be formally inducted during a ceremony at the NAE's annual meeting in Washington, D.C., on October 4, 2020.

\section{In Memoriam: Nigel Ricketts}

TMS extends its condolences to the family, friends, and colleagues of Nigel J. Ricketts, who passed away on June 29, 2019. A TMS member since 1992, he was actively involved in the Society's Aluminum Committee, serving as coorganizer of the Scandium Extraction and Use in Aluminum Alloys symposium at the TMS 2019 Annual Meeting \& Exhibition.
Throughout his career, Ricketts focused on steelmaking, base metals pyrometallurgy and hydrometallurgy, base metal flotation, magnesium and aluminum metallurgy, gold processing, and exotic metals, like vanadium and scandium. Most recently, he worked as the vice president of project and market development at Scandium International until June 2018. 


\section{David Williams Awarded RMS Honorary Fellowship}

Longtime TMS member and 1996

TMS Fellow David B. Williams was appointed Honorary Fellow by the Royal Microscopy Society (RMS). Williams, the Monte Ahuja Endowed Dean's Chair and Dean of the College of Engineering at The Ohio State University, was recognized for his pioneering work in developing analytical transmission electron microscopy (ATEM), as well as its applications to a broad range of materials. "Over the past 45 years his work has led to a new understanding of materials and microstructural evolution, including segregation, precipitation phenomena, phase diagrams, and phase transitions in metals and alloys," the RMS said of his appointment.

The distinction of Honorary Fellow is one of the most prestigious awards the society has to offer. The honors will be presented at the European Microscopy Congress 2020, to be held August 23-28, in Copenhagen, Denmark.

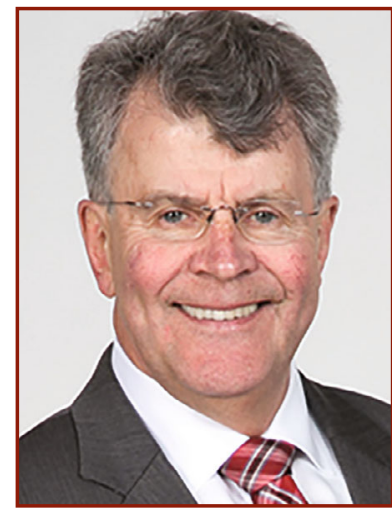

David B. Williams

\section{TMS Welcomes New Members}

\section{The TMS Board of Directors approved professional membership for the following individuals at its February 2020 meeting. Please join us in congratulating and welcoming them to all the privileges and benefits of TMS membership.}

\section{Abbasi Shirsavar, Mehran; United States \\ Abdul Wahab, Mohammad Azizol; Malaysia \\ Abdullah, Aboubakr M.; Qatar University, Qatar \\ Adam, Hamish; Boreal Laser, Canada \\ Adedayo, Babatunde Adelowo; National Iron Ore Mining Company Limited, Nigeria}

Ahmadi, Arezoo; RGQ, Iran

Ahnen, Violet M.; United States

Akhondi, Alireza; John R. Kieth, Australia

Akid, Robert; United Kingdom

Aliasghari, Hadi; Nano Shargh Abzar-e Toos Co., Iran

Aliof, Matt; United States

Allen, Brian; Dynamic System Inc. (Gleeble), United States

Allen, Harvey Pascoe; United Kingdom

Al-Shawi, Fadhil Abbas; United Kingdom

Altenbaugh, Derek; Robindale Energy Services, United States

Ambury, Rachael Fiona; United Kingdom

Anderson, Kevin; Brunswick-Mercury Marine, United States

Andrade, Marcio S.; IPEN, Brazil
Anstee, Richard Charles; Canada

Arai, Isao; Mitsubishi Materials, United States

Araneda, Eugenia; Chile

Aregawi, Wondwosen Abebe; University of Minnesota, United States

Asadikiya, Mohammad; Worcester Polytechnic Institute, United States

Baker, Lee; United Kingdom

Bansal, Anushka; United States

Barnes, Lee A.; United Kingdom

Basheer, Uday M.; United Kingdom

Bauerschlag, Nils; Hydro Aluminium Rolled Products $\mathrm{GmbH}$, Germany

Bechly, Maximilian; University of Applied Sciences Stralsund, Germany

Bennett, Charles Andrew; United Kingdom

Bertherat, Marc; Constellium, Switzerland

Besson, Jacques; École des Mines, France

Bezrukikh, Aleksandr I.; Siberian Federal University, Russian Federation

Bhaskar, Pragna; Georgia Institute of Technology, United States

Bhattacharya, Sudip; 6K Inc., United States

Bidari, Ehsan; Iran

Bilotti, Emiliano; United Kingdom
Bin Norizan, Mohd Natashah; Universiti Malaysia Perlis, Malaysia

Bittner, Benjamin; MeKo Laser Material Processing, France

Blackledge, Jonathan; United Kingdom

Blade, Lee; United Kingdom

Boff, James Charles; United Kingdom

Bojorquez, Francisco; Univerisdad de Sonora, Mexico

Boon, Jonathan Charles; United Kingdom

Boulaki, Foteini; United Kingdom

Bounou, Aikaterini; United Kingdom

Brandwood, Arthur; Australia

Breach, Christopher David; United Kingdom

Brismalein, David; Aluminium Dunkerque, France

Brown, Keith A.; Boston University, United States

Buller, Dane; United Kingdom

Buonocome, Giuseppe; United Kingdom

Burlatsky, Sergei; United Technologies Research Center, United States

Burton, Trevor; United Kingdom

Butala, Megan M.; University of Florida, United States

Buys, Ockert; United Kingdom 
Caine, Marcus; United Kingdom

Cairns, Daniel Lee; United Kingdom

Cam, Gurel; Iskenderun Technical University, Turkey

Campbell, David Stanley; United Kingdom

Campbell, Douglas James; United Kingdom

Campbell, Sylvia; United Kingdom

Carruthers, Alex William; University of Manchester, United Kingdom

Casali, Dick; Intel Corp., United States

Chakraborty, Kalyan Kumar; India

Chakraborty, Madhusudan; Adamas University, India

Chalmers, Frances Jane; United Kingdom

Chan, Helen M.; Lehigh University, United States

Channer, Akeel; NSWCCD, United States

Chen, Kongtao; University of Pennsylvania, United States

Chen, Peng; Texas A\&M University, United States

Chen, Yang-yuan D.; Institute of Physics, Academia Sinica, Taiwan

Cheng, Lin; University of Pittsburgh, United States

Chen-Wiegart, Yu-chen Karen; Stony Brook University/Brookhaven National Laboratory, United States

Chernetskiy, Ivan Vladimirovich; Ural Federal University, Russian Federation

Chico, Jonathan P.; Sandvik Coromant, Sweden

Choi, Sun; KIST, South Korea

Chowdhury, Sugata; National Institute of Standards and Technology, United States

Christensen, Steffen; Oman

Christopherson, Rhea; Materion, United States

Chui, Yin Tak; United Kingdom
Chung, Sheng-Heng; National Cheng Kung University, Taiwan

Clark, Julian Peter; United Kingdom

Clemmey, Richard Henry; United Kingdom

Co, Noelle C.; Blade Energy Partners, United States

Coley, Michael Delroy; University of the West Indies, Jamaica

Conway, Patrick; Jönköping University, Sweden

Cooper, Sarosh Sam; India

Cordill, Craig; Wagstaff, United States

Cortes, Pedro; Youngstown State University, United States

Costello, Kenneth J.; High Temp Measurement LLC, United States

Cote, Patrice; Rio Tinto Aluminium, Canada

Coury, Francisco Gil; Universidade Federal de São Carlos, Brazil

Couzinie, Jean-Philippe; Université Paris Est, France

Craig, Thomas Orr; United Kingdom

Cumings, John; University of Maryland, United States

Cunha, Jose; Alumar, Brazil

Dahlstrom, James; Gopher Resource, United States

Das, Hrishikesh; Pacific Northwest National Laboratory, United States

Davies, Peter J.; Germany

De Geuser, Frederic; Simap - Université Grenoble Alpes, France

De Palma, Alex C.; Univeristy of Texas Austin, United States

Dear, Felicity F.; Imperial College London, United Kingdom

Demetriou, Demetrios Haralambos; United Kingdom

Depan, Dilip; University of Louisianna Lafayette, United States

Deschênes, Jean-Michaël; Laserax, Canada

Devendhar Singh, Sanjay Kumar; Virginia Polytechnic Institute and State University, United States
Dienn, Henry; High-End Ltd., Japan

Dixit, Vikas; Intel Corp., United States

Doshi, Aakash; Almex USA Inc., United States

Douch, Colin J.; New Zealand

Dryden, Daniel M.; United States

Du, Chuanming; Tohoku University, Japan

Dykhuis, Andrew; United States

El-Atwani, Osman; Los Alamos National Laboratory, United States

Eliaz, Noam; Tel-Aviv University, Israel

Elliot, Christopher Neil; United Kingdom

Ellis, Elizabeth A.l.; Oak Ridge National Laboratory, United States

Emami Tabrizi, Isa; Sabanci University Orta Mahalle Tuzla, Turkey

Emdadi, Aliakbar; Germany

Eskil, Murat; Aksaray University, Turkey

Failla, David; Puget Sound Naval Shipyard, United States

Falconer, James Robert; United Kingdom

Fan, Zhe; Oak Ridge National Laboratory, United States

Fasoro, Abiodun; United States

Fayyad, Eman M.; Qatar University, Qatar

Fernandez-Silva, Beatriz; University of Sheffield, United Kingdom

Field, Kevin; University of Michigan, United States

Finstad, Terje G.; University of Oslo, Norway

Fleming, Thomas John; Ireland

Flowers, Patrick; Made In Space Inc., United States

Foster, Samuel Harry; United Kingdom

Fradet, Claude; Equibras, Canada 
Frankel, Gerald S.; The Ohio State University, United States

Fraser, Alex; Laserax Inc., Canada

Fukunaka, Yasuhiro; Kyoto University, Japan

Gambone, Justin J.; Georgia Institute of Technology, United States

Ganjkhanlou, Yadolah; University of Turin, Italy

Gao, Xu; Tohoku University, Japan

Gao, Youping; Castheon Inc., United States

Gao, Ziteng; United Kingdom

Geiss, Roy; Colorado State University, United States

Genin, Xavier; Solios Carbone, France

Gentils, Aurelie; Université ParisSaclay, France

Ghods, Masoud; Middle East Technical University, Turkey

Ghosh, Dipankar; Old Dominion University, United States

Giegerich, Larry Joseph; Canada

Gifford, Robert David; United Kingdom

Glowacka, Angelika; United Kingdom

Goken, Mathias; Friedrich-AlexanderUniversity Erlangen-Nürnberg, Germany

Gomez-Alvarez, Agustin; Univerisdad de Sonora, Mexico

Gopalan, Prashanth; Univeristy of Utah, United States

Gorain, Barun K.; Ore2Metal Inc., Canada

Goshi, Takuya; Denso International America Inc., United States

Greenwood, Sarah Catherine; United Kingdom

Griffin, Martin Stephen; United Kingdom

Groves, David; United Kingdom

Gu, Geun Ho; KAIST, South Korea

Guda Vishnu, Karthik; Purdue University, United States
Guiglionda, Gilles; Constellium CRV, France

Gurin, Elizabeth A.; Becton Dickinson, United States

Hackett, Benjamin; Texas A\&M University, United States

Han, Seung Min; KAIST, South Korea

Hanby, lan R.; New Caledonia

Harrington, Sean D.; United States

Harrington, Tyler; Oerlikon Metco, United States

Harrison, Marcus Elliot; United States

Hassan, Mohammad K.; Qatar University, Qatar

He, Jianhong; Oerlikon Metco, United States

$\mathrm{He}$, Jianjun; China

Heidarzadeh, Akbar; Shahid Madani University, Iran

Henshaw, David Cristopher; United Kingdom

Heo, Jungho; LS-Nikko Copper Inc., South Korea

Heydarinia, Ali; Iran

Hooshmand, Nasrin; Georgia Institute of Technology, United States

Hoover, Brian; Advanced Optical

Technologies, United States

Horn, Christopher; Ft. Wayne Metals Research/AMD, United States

Horrocks, Philip J.; United Kingdom

Hou, Wenyan; Central South University, China

Howard, Gary W.; Safety and Forensic Engineering, Canada

Howe, Tmothy Rowland; United Kingdom

Hughes, lan Gwyn; United Kingdom

Hunter, Graham C.; United Kingdom

Hunter, Luke William; United Kingdom

Igbafen, Akure Ohiomomo; Federal University of Technology, Nigeria

Ikeda, Satoshi; Nippon Light Metal Company Ltd., Japan
Isherwood, Patrick James M.; United Kingdom

Jacobson, Peter C.; Questek Innovations, United States

Jagannadham, Kasichainula; North Carolina State University, United States

Jahn, Matthias; University of Applied Sciences Stralsund, Germany

James, Richard; United Kingdom

Jamieson, Andrew; Navair, United States

Jermy, C A; United Kingdom

Jiao, Handong; Beijing Institute of Technology, China

Johnstone, James; United Kingdom

Jones, Nicholas G.; University of Cambridge, United Kingdom

Jones, Selwyn John Lloyd; United Kingdom

Joseph, David; United Kingdom

Joseph, Jithin; Deakin University, Australia

Jublot-Leclerc, Stephanie; JANNuS Université Paris-Saclay, France

Juwhari, Hassan; University of Jordan, Jordan

Kafexhiu, Fevzi; Institute of Metals and Technology, Slovenia

Kaligotla, Anand; Aludyne, United States

Kalra, Anisha; Indian Institute of Science Bangalore, India

Kantner, Chris; QuesTek Innovations, United States

Kartashov, Vadim V.; Ural Federal University, Russian Federation

Kawasaki, Megumi; Oregon State University, United States

Khalajhedayati, Amirhossein; TowerJazz, United States

Khan, Kamruzzaman; University of Michigan Ann Arbor, United States

Khoshghadam-Pireyousefan, Mohammad; Fardanegar, Iran 
Khosla, Nathan; United States

Kim, Sang-shik; Gyeongsang National University, South Korea

King, William L.; AK Steel Corp., United States

Kishore, Krishna Mugada; Indian Institute of Technology Delhi, India

Kocaefe, Duygu; University of Quebec at Chicoutimi, Canada

Kowathanakul, Nopasorn; United States

Krings, Daniel; Hydro Aluminium Rolled Products, Germany

Krishna, Athith; University of California, Santa Barbara, United States

Krishna, Vamsi; Univerisity of Chicago, United States

Kucza, Nikole J.; GE Global Research, United States

Kuhn, Erik; National Renewable Energy Laboratory, United States

Kuiken, Hilbrand; Quantillion Technologies, Netherlands

Kumar, M. Arul; Los Alamos National Laboratory, United States

Kurosaki, Ken; Osaka University, Japan

Lacey, Jeffery; Idaho National Laboratory, United States

Lahlouh, Bashar; University of Jordan, Jordan

Lam, Marcus Chunwai; MCAM, Monash University, Australia

Langille, Michael; Constellium Technology Center (C-TEC), France

Lannoy, Nate; United States

Lanzarotta, George; Kammerath \& Weiss, United States

Lau, Yang Hao; Institute of High Performance Computing, Singapore

Launiere, Cari; Argonne National Laboratory, United States

Lazarescu, Lucian; Technical University of Cluj-Napoca, Romania
Lee, Je In; Pusan National University, South Korea

Lee, Min-Ha; KITECH, South Korea

Leung, Chu Lun Alex; United Kingdom

Levine, Lyle E.; National Institute of Standards and Technology, United States

Li, Shunping; Apple, United States

$\mathrm{Li}$, Xiangguo; University of California, San Diego, United States

Liao, Michael E.; University of California, Los Angeles, United States

Lim, Chao Voon Samuel; Monash University, Australia

Lips, Andor; Netherlands

Liu, Jing; University of Alberta, Canada

Liu, Xianbin; Singapore

Long, Gerard Christopher; United Kingdom

Longwell, David Jame; United Kingdom

Ludwig, Alfred; Ruhr-University Bochum, Germany

Lukac, Frantisek; Institute of Plasma Physics of the Czech Academy of Sciences, Czech Republic

Luo, Yan; University of Science \& Technology Beijing, China

Lyle, Luke A.M.; Carnegie Mellon University, United States

$\mathrm{Ma}, \mathrm{Ke}$; University of Connecticut, United States

Mackie, David Murray; United Kingdom

Macklin, Stephen Robert; Australia

Maddox, Jennie C.; Mississippi State University, United States

Mahieu, Pierre; Solios Carbone, France

Makepeace, Jeremy D.; United Kingdom

Martin Da Silva, Iva Luisa; United Kingdom

Marvel, Christopher J.; Lehigh University, United States

Maxwell, Austin; Alcoa, Australia

Mayandi, Jeyanthinath; SMN, Department of Physics, Norway

McGinnity, Brian T.; United Kingdom
McGregor, Ronald; Canada

McLaughlin, Paul; United Kingdom

McQueen, Fraser Wilson; United Kingdom

Meadley, Philip Frank; Singapore

Meddeb, Sami; Grenoble Institute of Technology, France

Mei, Jun; Queensland University of Technology, Australia

Mendieta, Marla J.; Ormco, United States

Mendoza-Cruz, Ruben; University of Texas at San Antonio, United States

Menze, Roman; MeKo Laser Material Processing, Germany

Mermet, Stéphane; Liberty Aluminium Dunkerque, France

Mhay, Amandeep Singh; United Kingdom

Miao, Jiashi; The Ohio State University, United States

Mihalop, Owen Daniel; United Kingdom

Milhet, Xavier; Prime Institute CNRS ENSMA, France

Millar, Dean Lee; Canada

Mirabedini, Pegah S.; University of California, Riverside, United States

Mirak, Mohammad; Behine Sanjesh Pars Alma, Iran

Mitkova, Maria; Boise State University, United States

Mohaghegh Moein, Alireza; Iran

Mohammadzadeh, Ahad; Iran

Molaei, Fatemeh; University of Arizona, United States

Mollah, Shahab; University of South Carolina, United States

Monaghan, Elizabeth A.; GE Power, United States

Mondal, Kunal; Idaho National Laboratory, United States

Moon, Yun Sung; South Korea

Moore, Richie; United Kingdom 
Moravcik, Igor; Brno University of Technology, Czech Republic

Moseley, Steven Glyn; Liechtenstein

Moss, Allan; Canada

Mukhtarov, Shamil Khamzaevich; Institute for Metals Superplasticity Problems, Russian Federation

Mulheron, Michael John; United Kingdom

Munoz, Jorge A.; University of Texas at El Paso, United States

Muta, Hiroaki; Osaka University, Japan

Mutreja, Isha; University of Minnesota, United States

$\mathrm{Na}$, Young-Sang; Korea Institute of Materials Science, South Korea

Naeimi Panjaki, Alireza Ali; Japan

Naji, Hojjat; I.T. Forging Co., Iran

Nam, SungWoo; University of Illinois Urbana-Champaign, United States

Nasiri Khalil Abad, Sajjad; Sahand University of Technology, Iran

Nasouri, Reza; University of Texas at San Antonio, United States

Nelaturu, Phalgun; University of Wisconsin, United States

Nicholls, Peter; United Kingdom

Niitsu Campo, Kaio; Unicamp, Brazil

Nish, John; Welman Dynamics, United States

Nordlund, Kai; University of Helsinki, Finland

Oba, Satoshi; Nippon Light Metal Company Ltd., Japan

O'Connor, Christopher; United Kingdom

Ogle, Richard; United Kingdom

Oh, Hyunseok; Massachusetts Institute of Technology, United States

O'Hara, Dante J.; Naval Research Laboratory, United States

Ohishi, Yuji; Osaka University, Japan

Orr, Jessica; University of Dayton Research Institute, United States
Osei-Agyemang, Eric; Lehigh University, United States

Overman, Nicole R.; Pacific Northwest National Laboratory, United States

Owusu-Konadu, Barbara M.; BOK Engineering Consulting Services, Ghana

Ozagir, Ozcan; United Kingdom

Pandolfelli, Victor; Alcoa Laboratory, Federal University of Sao Carlos, Brazil

Papaj, Ewa; United Kingdom

Papakonstantinou, Konstantinos; United Kingdom

Papanikolaou, Michail; Cranfield University, United Kingdom

Parisi, Cristian; United Kingdom

Park, Jonghyun; Missouri University of Science and Technology, United States

Park, Joo Hyun; Hanyang University, South Korea

Park, Joon Young; Harvard University, United States

Park, Yongmin; KG Dongbu Steel, South Korea

Parra, Roberto A.; Universidad de Concepcion, Chile

Parra-Sanchez, Victor Roberto; Universidad de Concepcion, Chile

Pateras, Anastasios; Los Alamos National Laboratory, United States

Pavlina, Erik J.; AK Steel Corporation, United States

Pearson, Alastair Scott; United Kingdom

Pecharsky, Vitalij K.; lowa State University, United States

Pedroli, Herve; Aluminium Dunkerque, France

Perry, Carole; Nottingham Trent University, United Kingdom

Phanopoulos, Christopher; Belgium

Pickering, Ed; University of Manchester, United Kingdom

Pierson, Ed; Lockheed Martin Space, United States

Ping, Xue; Jianghan University, China
Ponce, Arturo; University of Texas at San Antonio, United States

Popplewell, Guy; United Kingdom

Porter, Matthew; United Kingdom

Potter, Michael; RJ Lee Group, United States

Potter, Tara Jessica; United Kingdom

Pouladi, Sara; University of Houston, United States

Pouranvari, Majid; Sharif University of Technology, Iran

Pramanik, Brahmananda; Montana Tech, United States

Prasetyo, Erik; Indonesian Institute of Sciences, Indonesia

Priddy, Matthew W.; Mississippi State University, United States

Primeau, Pierre J.A.; Golder Associates Ltd., Canada

Qu, Jun; Oak Ridge National Laboratory, United States

Raabe, Dierk R.; Max-Planck Institute, Germany

Rack, Alexander; European Synchrotron Radiation Facility, France

Ramasagara Nagarajan, Varun; Altair ProductDesign, United States

Rao, Apparao M.; Clemson University, United States

Rashad, Mohamed; Central Metallurgical Research and Development Institute, Egypt

Rashed, Md Golam; Australia

Rasooli, Novin; University of Tehran, Iran

Rathkanthiwar, Shashwat; Indian Institute of Science Bangalore, India

Rawal, Suraj P.; Lockheed Martin Space, United States

Ray, Atish K.; Novelis Inc., United States

Raymond, Paul; United Kingdom

Razmi, Jafar; Arizona State University, United States 
Rees, David Tien; University College London, United Kingdom

Ren, Qiang; University of Science and Technology Beijing, China

Rhinehart, Katherine; United States

Richard, Gerald; Magma Foundry Technologies Inc., United States

Roma, Guido; CEA, France

Rosefort, Marcel; Trimet Aluminium SE, Germany

Roy, Sougata; Oak Ridge National Laboratory, United States

Sabarudin, Ahmad; Malaysia

Sabeti Monfared, Ahad; Iran

Sadawy, Mosaad Mohamad; AlAzhar University, Egypt

Sadeghi, Nima; Sahand University of Technology, Iran

Salas Mula, Daniel; Texas A\&M University, United States

Sanchez-Corrales, Victor M.; University of Sonora, Mexico

Sandnes, Espen; Norwegian University of Science and Technology, Norway

Sarkar, Soumalya; United Technology Research Center, United States

Schaffer, Jeremy; Fort Wayne Metals, United States

Scheller, Piotr R.; TU Begakademie Freiberg, Germany

Schellert, Steven; Universitat Siegen, Germany

Schliephake, Daniel; Monash Centre for Additive Manufacturing, Australia

Schofield, Timothy Robert; United Kingdom

Scholtz, Juliane; The University of Michigan, United States

Schrefl, Thomas; Danube University Krems, Austria

Schriner, Doug; North American Stainless, United States
Schuck, Christopher F.; University of Delaware, United States

Schulz, Wencke; Bundesanstalt fur Materialforschung und -prufung, Germany

Scott, James; United Kingdom

Seetharaman, Sankaranarayanan; ANSYS, India

Sengupta, Debasis; United States

Shakoor, Abdul; Qatar University, Qatar

Shamlaye, Karl; Deakin University, Australia

Shapiro, Alexander E.; Titanium Brazing Inc., United States

Shellam, Richard; United Kingdom

Shen, Xian Chun; CITIC International Cooperation Co. Ltd., China

Shepherd, Nigel; University of North Texas, United States

Sherman, Andrew J.; Powdermet Inc./ Terves Inc., United States

Shibayama, Atsushi; Akita University, Japan

Shimokawa, Tomotsugu; Kanazawa University, Japan

Shin, Seungha; University of Tennessee, United States

Shinozaki, Maya; China

Shokri, Nayer; Sahand University of Technology, Iran

Shun, Tao-Tsung; Feng Chia University, Taiwan

Singh, Ankit; Welspun Corp. Ltd., India

Singh, Harminder; Guru Nanak Dev University, India

Sismondi, Shawn; United Kingdom

Sliem, Mostafa Hussien; Qatar University, Qatar

Smith, David John; United Kingdom

Softly, Tilly; United Kingdom

Spark, Caroline; United Kingdom

Srinivasan, Srilok; Argonne National Laboratory, United States
Stamboulis, Artemis; University of Birmingham, United Kingdom

Stanley, Ashlynn M.; NAVAIR, United States

Steglich, Jan; TRIMET Aluminium SE, Germany

Steinbach, Sonja; DLR, Germany

Stephens, Alan; United Kingdom

Stevens, Wayne; United Kingdom

Strawbridge, Anna; United Kingdom

Su, Yanqing; University of California, Santa Barbara, United States

Sun, Weiping; Nucor Corporation, United States

Sutton, Yvonne; United Kingdom

Szczepanski, Christopher; Special Metals Corp., United States

Taghavimehr, Mehrnoosh; lowa State University, United States

Talapatra, Anjana Anu; Los Alamos National Laboratory, United States

Tan, Pengfu; Glencore, Switzerland

Tang, Fengzai; University of Warrick, United Kingdom

Tang, Wei; Ames Laboratories, United States

Tariq, Hanan Abureh; Qatar University, Qatar

Taylor, Justin Paul; Hong Kong

Thompson, Vicki; Idaho National Laboratory, United States

Todoroki, Hidekazu; Japan

Toh, Qiuyi; United Kingdom

Tomlinson, Sarah Louise; United Kingdom

Tong, Jianhua; Clemson University, United States

Torres, Jonathan; Bucknell University, United States

Torres Arango, Maria; Brookhaven National Laboratory, United States 
Trevino, Diana A.; Worley, United States

Trivedi, Pankaj B.; Booz Allen Hamilton, United States

Trotter, Carolyn; BlueScope Steel Ltd., Australia

Tsai, Ming-Hung; National Chung Hsing University, Taiwan

Ueda, Shigeru; Tohoku University, Japan

Ulrich, Tashiema L.; United States

Unnikrishnan, Vinu; West Texas A\&M University, United States

Uwidia, Ita E.; University of Benin, Nigeria

verma, Narendra Kumar; India

Walsh, Brian; United Kingdom

Walther, Frank; TU Dortmund University, Germany

Wang, Kang; University of Virginia, United States

Wang, Liancheng; Central South University, China

Wang, Xin; University of California, Irvine, United States

Wang, Yan; University of Nevada Reno, United States

Wang, Yongjie; University of California, Berkeley, United States

Wang, Yuechen; Nano and Advanced Materials Institute Limited, Hong Kong

Ward, Al; United Kingdom

Ward, Richard Wiltshire; United Kingdom

Waterton, Michael; United States
Watson, Frank; Safe Labs, United States

Wen, Wei; ATI Specialty Materials, United States

Werner, Andreas; United Kingdom

Weston, Nicholas Samuel; University of Sheffield, United Kingdom

Whitaker, lain Robert; United Kingdom

Whiteman, Dean; Alcoa, Australia

Williams, Adrienne D.; ADee

Technologies LLC, United States

Williams, Maureen; National Institute of Standards and Technology, United States

Williams, Nigel R.; United Kingdom

Wilson, Orla; John Hopkins University, United States

Wilson, Paul; Boeing, United States

Wint, Natalie; United Kingdom

Winter, Thomas; Naval Nuclear Laboratory, United States

Wolff, Sarah J.; Texas A\&M University, United States

Wolfrum, Ed; National Renewable Energy Laboratory, United States

Wongsa-Ngam, Jittraporn; King Mongkut's Institute of Technology Ladkrabang, Thailand

Woodfill, James C.; United States

Wu, Jyh-Ming; National Tsing Hua University, Taiwan

Wyatt, Keith; United Kingdom

Xenos, Epameinondas; Elval Halcor S.A., Greece

Xiong, Frank F:; Heaptech, United States

$\mathrm{Xu}$, Changxue; Texas Tech University, United States
Xu, Xin; Imperial College London, United Kingdom

Yamaguchi, Katsunori; Waseda University, Japan

Yang, Judith; University of Pittsburgh, United States

Yarnall, John Thomas; United Kingdom

Young, George; United States

Yury, Chumlyakov Ivanovitch; Tomsk State University, Russian Federation

Yuryev, Pavel O.; Siberian Federal University, Russian Federation

Zaid, Hicham; University of California, Los Angeles, United States

Zaldivar Escola, Facundo; LHD, Argentina

Zamborszky, Ferenc; MagnetecUngarn Kft., Hungary

Zangari, Giovanni; University of Virginia, United States

Zhang, Hao; University of Alberta, Canada

Zhang, Yan; United Kingdom

Zhao, Huan; Max-Planck-Institut für Eisenforschung $\mathrm{GmbH}$, Germany

Zheng, Leixia; Central South University, China

Zhu, Jun; Sanhua Texas Technology Center, United States

Zhu, Zhongping; China

Zuback, James; Pennsylvania State University, United States

Zuo, Jian Min J.; University of Illinois, United States

*Membership grade recommendations are based on a review of credentials provided by the individuals. These credentials are taken on the honor system and not independently verified except by exception. 